\title{
Endoscopic Excision of Solitary Neurofibroma Arising from Posterior Nasal Septum
}

\author{
${ }^{1}$ Parvinderjit Singh Kohli, ${ }^{2}$ Kanu Goel \\ ${ }^{1}$ Consultant, Department of ENT and Head and Neck Surgery, Columbia Asia Hospital, Patiala, Punjab, India \\ ${ }^{2}$ Consultant, Department of Pathology, Columbia Asia Hospital, Patiala, Punjab, India
}

Correspondence: Parvinderjit Singh Kohli, Consultant, Department of ENT and Head and Neck Surgery, \#26/1-A, Azad Nagar Sirhind Road, Patiala, Punjab, India, P hone: +91-98153-90718, e-mail: parrykohli@ yahoo.com

\begin{abstract}
Neurogenic tumors form a very small percentage of all neoplastic lesions of the head and neck region. However, the head and neck region is by far the most common location for benign peripheral neural tumors. Several cases involving the nasal cavity and paranasal sinuses have been sporadically documented throughout the medical literature. We present a rare case of a solitary neurofibroma arising from the posterior nasal septum of a 37-year-old woman. En bloc surgical resection of the mass was achieved by endoscopic approach. This case highlights the importance of considering this clinical entity in the differential diagnosis when encountering a unilateral soft tissue mass in the nasal cavity and use of endoscopic technique in the management of such tumors.

Abbreviations: Contrast enhanced computed tomography (CECT), Magnetic resonance imaging (MRI).

Keywords: Benign peripheral nerve sheath tumors, Neurofibroma, Endoscopic endonasal approach.
\end{abstract}

\section{INTRODUCTION}

Peripheral nerve sheath tumors arise from both the Schwann cells and the perineural cells. ${ }^{1}$ Either or both of these cell types probably give rise to neoplasms that are included in the diagnostic category of peripheral nerve sheath tumors. These tumors are classified as benign and malignant. B enign tumors include neurofibroma and solid schwannoma (neurilemmoma, neurilemoma, neurinoma). Malignant variant includes neurogenic sarcoma (malignant schwannoma, malignant neurilemoma, malignant neurinoma, malignant nerve sheath tumor, neurogenic schwannoma and neurofibrosarcoma). A bout 25 to $45 \%$ of neurofibromas occur in the head and neck region and only $4 \%$ involve the nasal cavity and paranasal sinuses. ${ }^{2}$ Neurofibroma manifests as an isolate lesion or multiple tumors, as it happens in type 1 neurofibromatosis or von Recklinghausen's disease. The potential for malignant transformation is of $2.6 \%$, and in the syndrome, it varies between 3 and $15 \%{ }^{3}$

Cases of benign peripheral nerve sheath tumors involving the nose have been sporadically documented throughout the medical literature. We present a rare case of solitary neurofibroma arising from the posterior nasal septum in a 37-year-old woman, which was endoscopically excised.

\section{CASE REPORT}

A 37-year-old female presented to the outpatient department of our hospital with complaints of foul smelling left nasal discharge and nasal obstruction for last two years. Nasal discharge was mucopurulent and persistent throughout the year; decreasing in consistency with course of antibiotics. $\mathrm{N}$ asal obstruction was unilateral mainly on left side, which had progressed to be permanent on presentation. There had been two episodes of epistaxis from left side; spontaneous onset and cessation after sometime. Postnasal drip was present. There was no history of pain, toothache, anosmia, visual symptoms and symptoms suggesting of any intracranial pathology. Anterior rhinoscopic examination revealed mucopurulent discharge in the left nasal cavity. Clearing the nasal cavity of secretions revealed soft tissue mass in the posterior part of left nasal cavity. $\mathrm{N}$ asal endoscopic examination was done under local anesthesia. Examination revealed smooth mucosa covered polypoidal mass in the posterior part of left nasal cavity. This mass was pushing the middle turbinate laterally and had attachment with the nasal septum on a broad base (Fig. 1). Nasopharynx was free of any extension of mass. CECT nose and PNS were done to know about the exact size of the mass and site of origin. CT revealed slightly contrast enhancing soft tissue mass in the posterior part of nasal cavity extending till posterior choana, pushing and eroding 


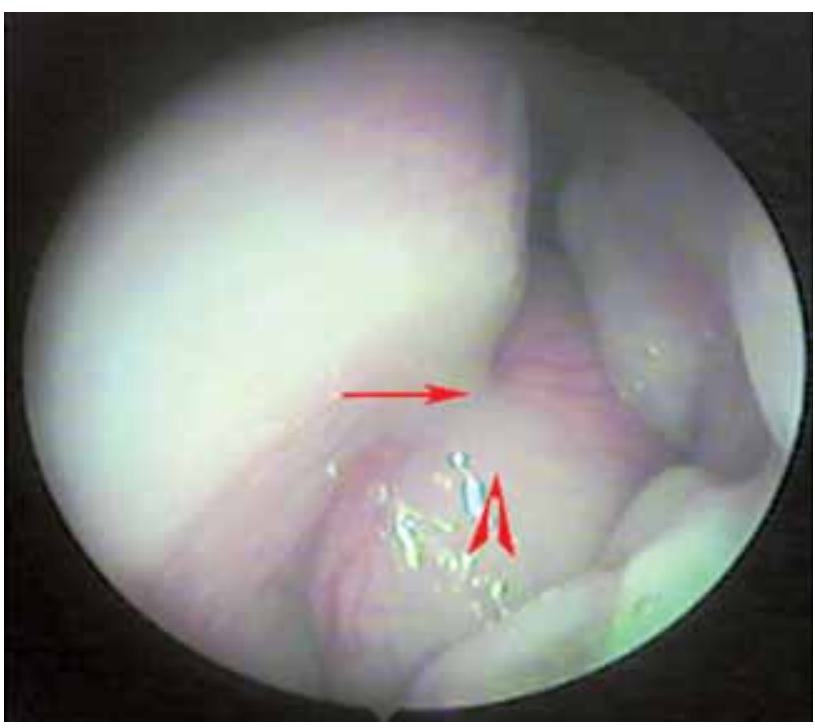

Fig. 1: Preoperative photograph of the left nasal cavity taken through $0^{\circ}$ nasal endoscope showing smooth, mucosa covered mass (arrowhead) attached to the septum (arrow)

the bony nasal septum anterior to sphenoid rostrum and retained secretions in left maxillary sinus ( $F$ igs $2 A$ and $B$ ). On nasal endoscopic examination and CT scan, the tumor seemed to be of benign nature and arising from the nasal septum. The patient was taken up for endoscopic endonasal excisional biopsy of the tumor under general anesthesia. The tumor was excised into taking margins of adjacent septal mucosa, which created a septal defect at the excision site. The tumor did not bleed much during excision. Examination of the mass after excision revealed it to be of friable nature, measuring approximately $4 \times 4 \mathrm{~cm}$ and cut section showing gelatinous consistency (Fig. 3). Histopathological examination of the tumor revealed it to be a benign peripheral nerve sheath tumor; neurofibroma. Hematoxylin and eosin staining showed ovoid to spindle shaped cells of fairly uniform size and shape with no abnormal mitotic activity, but, abundant pale staining myxoid connective tissue. One year into follow-up, the patient is free of any recurrences; with a septal defect in the posterior part of nasal septum causing no morbidity (Fig. 4).

\section{DISCUSSION}

Neurofibroma is a benign peripheral nerve sheath tumor occurring rarely in the nasal cavity, paranasal sinuses and nasopharynx. There is no sex predilection for neurofibromas and they commonly occur in the third or fourth decade of life. Solitary neurofibroma arises along a nerve trunk, occurring as a spontaneous lesion. The tumors are frequently centrally located, and are usually nontender. The definitive neural source for a neurofibroma arising from the nasal septum is generally difficult to establish. It may originate

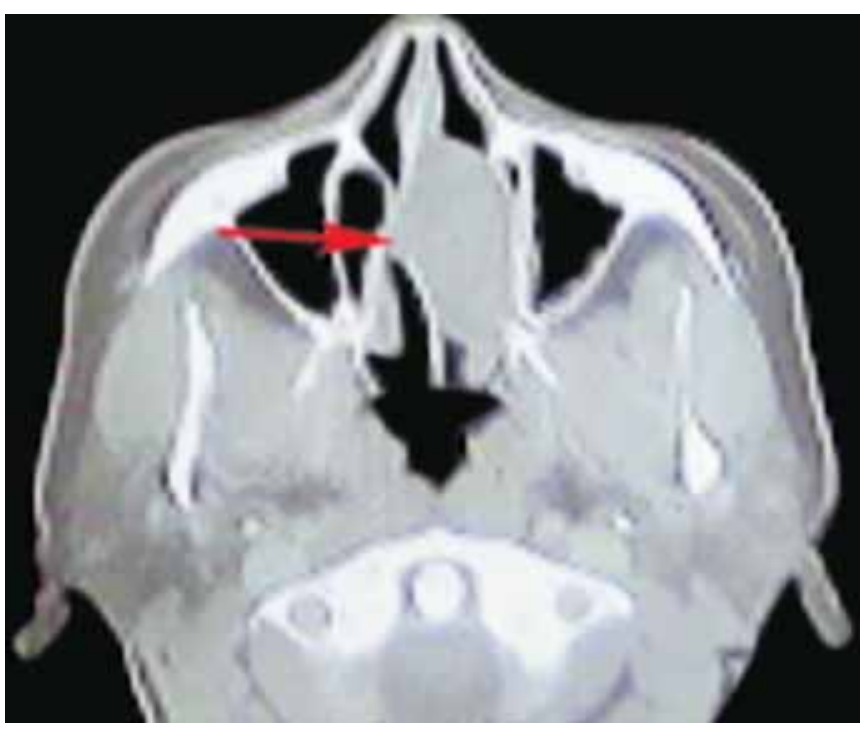

Fig. 2A: Preoperative contrast-enhanced CT scan of the patient (axial cut) showing minimally contrast enhancing soft tissue mass in the left nasal cavity extending till posterior choana with erosion of the bony nasal septum (arrow)

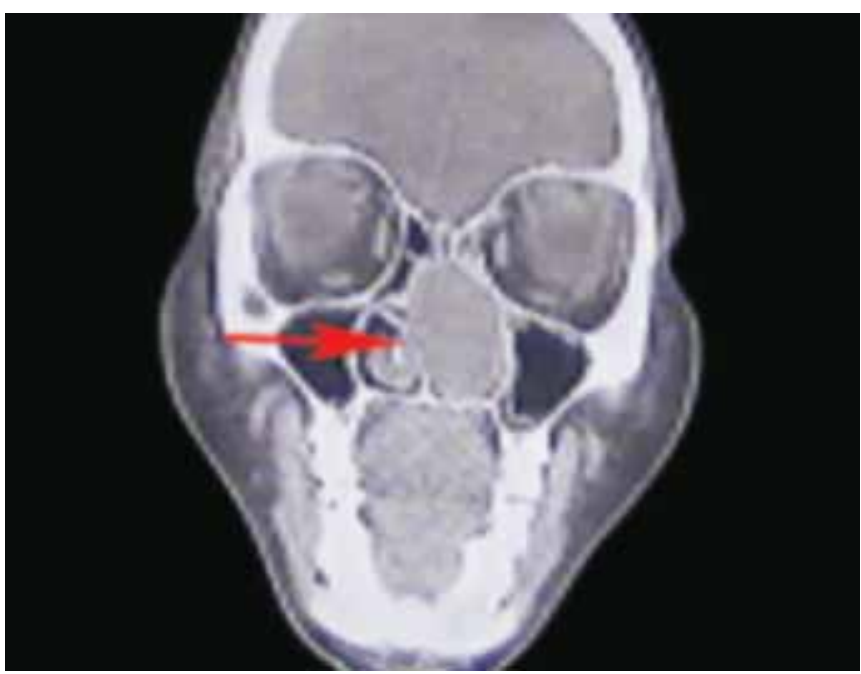

Fig. 2B: P reoperative contrast-enhanced CT scan of the patient (coronal cut) showing minimally contrast enhancing mass in the left nasal cavity completely filling it with erosion of the bony nasal septum (arrow)

from autonomic fibers reaching the septum via the greater palatine nerve, from the anterior ethmoidal nerve, which innervates the anterior part of the septum or from the nasopalatine nerve, which supplies the posterior part of the septum. ${ }^{4}$

Only a relatively small number of Schwann cell tumors including neurofibromas, involving upper respiratory system have been reported in literature. In most papers, only one or two cases are described. ${ }^{5-8}$ Signs and symptoms depend on the anatomic site involved, the nerve of origin and the extent of the tumor. Patients may complain of unilateral nasal obstruction, epistaxis, mucopurul ent rhinorrhea, anosmia, facial swelling, proptosis and pain when the maxillary sinus 


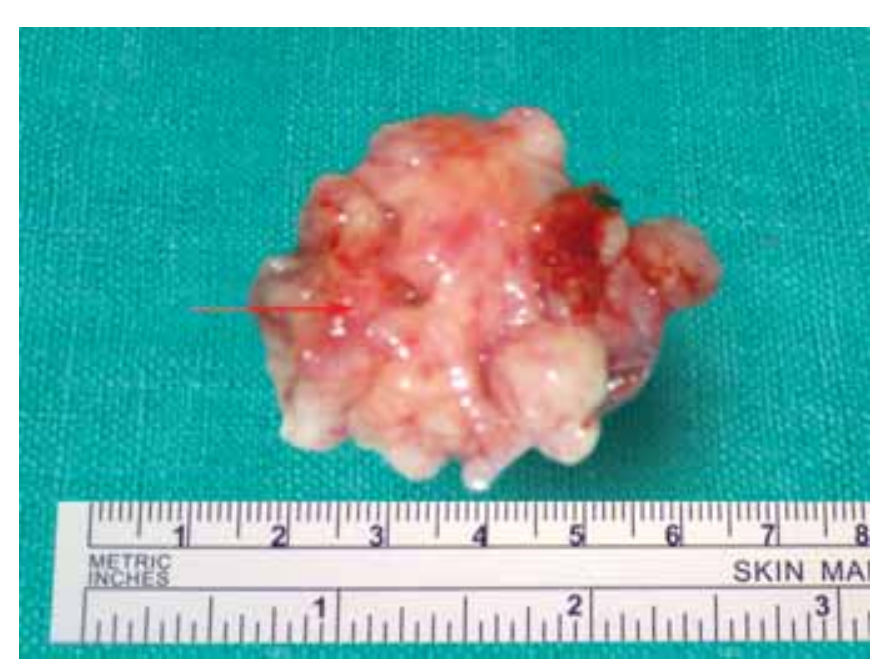

Fig. 3: The excised tumor (cut section) showing gelatinous central part of the tumor (arrow)

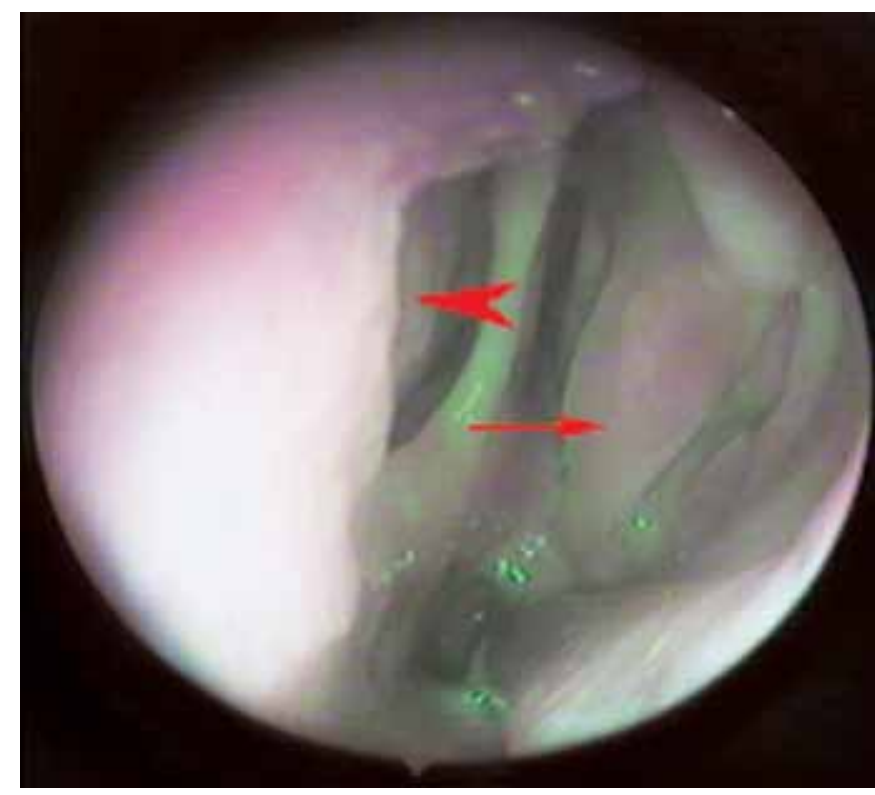

Fig. 4: Postoperative photograph of the left nasal cavity taken through $0^{\circ}$ nasal endoscope showing septal defect (arrowhead) at the site of excision of the tumor and no evidence of residual/ recurrence. Middle turbinate is shown by arrow

is involved. Sphenoid sinus involvement may lead to cranial nerve palsy, deep retro-orbital pain and occipital, frontal and temporal headache. These clinical problems are similar to those produced by other nonepithelial neoplasms involving this area. Because of the nonspecific nature of these signs and symptoms, the diagnosis of neurofibroma usually is made only when the lesion is biopsied or excised. In patients who have von Recklinghausen's disease, the diagnosis of neurofibroma is suggested when a mass lesion arises in this area.

Solitary and multiple neurofibromas have the same microscopic features. They contain spindle shaped cells with fusiform or wavy nuclei found in a delicate connective tissue matrix; this matrix may be notably myxoid in character.
These lesions may be well circumscribed or may blend into surrounding connective tissue. Mast cells are characteristically scattered throughout the lesion. A histologic subtype known as plexiform neurofibroma is regarded as being highly characteristic of neurofibromatosis. ${ }^{1}$ Immunohistochemically, neurofibroma shows immunoreactivity of S-100 protein, neuron specific enolase (NSE) and vimentin but not for desmin or smooth muscle actin, which may be useful in differentiating between neurofibroma and other tumors. ${ }^{3}$

In most cases, the pathologist will not experience difficulty in making the diagnosis of a neurofibroma or a benign schwannoma because these tumors do have a distinctive histologic appearance. Some problems may be encountered, however in differentiating between these two lesions, especially in small biopsy or curettage specimens. Schwannomas have a capsule, neurofibromas generally do not. The myxoid changes as well as the highly cellular areas found in schwannomas may occur also in neurofibromas, although nuclear palisading usually is not a prominent feature in neurofibromas. Thus, these two neoplasms do exhibit overlapping histologic features. Since malignant change may develop in neurofibromas but not in schwannomas, the pathologist should attempt to differentiate between these tumors. ${ }^{9}$ In nose and paranasal sinuses, neurofibromas with myxoid changes may be difficult to differentiate from the myxomas that involve facial bones. Neurofibromas should be differentiated from neoplasms derived from fibroblasts (fibromatosis, fibrosarcoma); fibrous histiocytoma; and from osseus and fibro-osseus lesions (fibrous dysplasia, ossifying fibroma, osteosarcoma). ${ }^{9}$

When arising in the nose and paranasal sinuses, neurofibromas may cause bone erosion, as demonstrated in Figs $2 \mathrm{~A}$ and $\mathrm{B}$. Some neurofibromas grow by expansion, and thus can erode the adjacent bone by pressure. Some can infiltrate locally, even into bone, and can lead directly to bone resorption. Thus, bone erosion does not necessarily indicate the presence of a malignant lesion.

High-resolution CT scanning in various projections is thought to be the best imaging investigation for neurofibromas. Preoperative evaluation of $\mathrm{CT}$, however, allow us to reveal the pushing borders of the tumors, rather than the invasive character of malignancy. M RI with gadolinium contrast is indicated in areas with intraorbital or intracranial extension, and for more exact delineation of the tumor from the normal soft tissues. ${ }^{10} \mathrm{~F}$ urthermore, M R I is helpful in differentiating the neoplasm from retained secretions or inflammatory changes within the sinuses and nasal cavity. 
Surgical resection is the treatment of choice for sinonasal neurofibromas. An adequate procedure depends on tumor extension, and includes variety of external and endonasal approaches. When resecting sinonasal neurofibromas, radical surgical resection probably is not required, and functional and cosmetic considerations should be taken into account. In recent years, most frequently, broad range of benign tumors of nasal cavities and paranasal sinuses are being managed endoscopically.Transnasal endoscopic resection is useful if the neurofibroma is solitary and located in the nasal cavity and the origin can be identified. Some authors have published very good outcomes after endoscopic surgery with no signs of recurrence. ${ }^{7,8}$ It is important to visualize the origin of the tumor, which allows macroscopic complete resection under endoscopy. However, if the operative findings make it impossible to excise the neoplasm completely by endoscopic means, the surgeon should be prepared to convert to open surgical procedures. If not excised completely, neurofibromas may recur locally and require further local resection. Recurrence of this tumor is rather rare, although more common than schwannoma.

\section{CONCLUSION}

Isolated benign lesions of the nasal cavity, including very rare tumors like those presented here, can be safely approached and removed endoscopically.

\section{REFERENCES}

1. Batsakis J. Tumors of the head and neck. Clinical and pathological consideration (2nd ed). Baltimore: W illiams and Wilkins 1979;313-33.

2. Souza L, Oliveira J, F reitas $M$, et al. N eurofibroma paciniano: Relato de um caso raro de localizacao itraoral. Rev Brasil Otorhinolaringol 2003;69:851-54.

3. Silva V, A gra L, Freitas $M$, et al. Neurofibroma nasal: Relato de caso e revisao de literature. Rev Brasil Otorhinolaringol 1999;65:172-74.

4. RobitailleY, Seemayer TA, EI D eiry A. Peripheral nervetumors involving paranasal sinuses and nasopharynx. Cancer 1975;35:1254-58.

5. A garwal M K, Gupta OP, Samant HC, Gupta S. Neurofibroma of the maxillary antrum. Oral Surg 1979;48:150-52.

6. A nnino DJ (J r), Domanowski GF, V aughan CW. A rare cause of nasal obstruction: A solitary neurofibroma. Otolaryngology Head and Neck Surgery 1991;104:484-88.

7. Hirao M, Gushiken T, Imokawa H, Kawai S, Inaba H, T sukuda $M$. Solitary neurofibroma of the nasal cavity: Resection with endoscopic surgery. J Laryngol Otol 2001;115(12):1012-14.

8. Y ong DK, Chang HB , J ang SS, K ei W S. Transnasal endoscopic excision of an isolated neurofibroma of the nasal septum. Rhinology 1997;35:89-91.

9. K arl H Perzin, Howard Pangu, Steven W echter. Nonepithelial tumors of the nasal cavity, paranasal sinuses and nasopharynx. A clinicopathologic study. XII: Schwann cell tumors (Neurilemoma, N eurofibroma, M alignant Schwannoma). Cancer 1982;50:2193-202.

10. El-Saggan A, O lofsson J, K rossner B. Sinonasal schwannoma: Two case reports and review of literature. International Congress Series 2003;1240:503-07. 